\title{
Performances morpho-physiologiques et agronomiques du niébé [Vigna unguiculata (L.) Walpers] en conditions du déficit hydrique
}

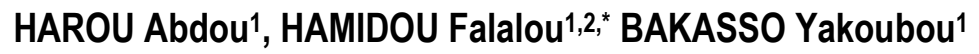 \\ 1 : Département de biologie, Faculté des Sciences et Techniques, Université Abdou Moumouni, B.P. 10662, Niamey- \\ Niger \\ 2 : Institut International de Recherche sur les Cultures des zones Tropicales et Semi-Arides (ICRISAT), Centre \\ Sahélien, B.P. 12404, Niamey-Niger \\ * : Auteur pour correspondance, Email : h.falalou@cgiar.org
}

Original submitted in on 23rd March 2018. Published online at www.m.elewa.org on $31^{\text {st }}$ August 2018 https://dx.doi.org/10.4314/jab.v128i1.1

\section{RESUME}

Objectif : Ce travail vise à étudier les effets du déficit hydrique intermittent et de la saison de la culture sur certains paramètres morpho-physiologiques et agronomiques à travers une évaluation de son impact sur l'indice de la surface foliaire, la surface foliaire, la biomasse des feuilles et le rendement en graines.

Méthodes et résultats : Dix-huit variétés de niébé ont été testés au champ en saisons froide et chaude. Deux traitements hydriques qui sont bien irrigué (BI) et déficit hydrique intermittent $(\mathrm{DH})$ couvrant les stades végétatif et reproductif ont été étudiés. Nos résultats ont montré que la date de $50 \%$ floraison a été allongée de 5 jours en moyenne en saison froide sous l'effet du déficit hydrique. L'indice de surface foliaire (LAI), la surface foliaire et la biomasse des feuilles ont été plus élevés en conditions $\mathrm{BI}$. Le DH a significativement réduit le rendement graines de toutes les variétés $(P<0,001)$. La baisse du rendement en graines est de $88 \%$ en saison froide contre $67 \%$ en saison chaude. L'étude a révélé que les variétés KVX-61-1, IT96D-610 et IT98K-1111-1 sont les plus performantes pour les deux régimes hydrique en termes du rendement.

Conclusion et application : L'identification des variétés précoces et/ou tolérantes à la sécheresse intermittente pouvant améliorer la culture du niébé au stade végétatif et/ou reproductif pourrait permettre d'augmenter le rendement et affecter la production de cette culture au Niger.

Mots clés : Déficit hydrique, Niébé, rendement graines, biomasse, physiologie

\section{ABSTRACT}

Objective : the aim is to investigate the intermittent drought stress and the season effects on agro-physiological parameters by assessing its impact on the leaf area index (LAl), leaf area, leaf dry weight and grain yield. Methods and Results : Eighteen cowpea genotypes were tested in the field during cold and hot seasons. Two water treatments, well-watered (WW) and intermittent drought (WS), were imposed at vegetative and reproductive stages during three experiments. Our findings showed that the flowering date delayed up to 5 days in the cold season under water deficit. The LAl, leaf area and leaf dry weight were high under WW conditions. Under $\mathrm{DH}$, grain yield decrease significantly $(\mathrm{P}<0.001)$ up to 88 and $67 \%$ in the cold and hot seasons respectively. KVX-61-1, IT96D-610 and IT98K-1111-1 showed the highest grain yield under both seasons and $r$ both water treatments. 
Conclusion and application : The identification of early and/or intermittent drought tolerant varieties that may improve cowpea cultivation at the vegetative and / or reproductive stages could lead to increase grain yield and production of cowpea in Niger.

Keywords : Water deficit, cowpea, grain yield, physiology, biomass

\section{INTRODUCTION}

Depuis la fin des années 1960, les régions sahéliennes subissent une sécheresse due à une importante baisse de la pluviométrie et à une augmentation de sa variabilité spatiotemporelle (Nana et al., 2009). Dans cette région où la culture pluviale est très largement majoritaire, la sécheresse est une contrainte permanente de la production agricole (Clavel et al., 2005). C'est ce qui explique largement la faiblesse des rendements et les fortes variations de la production agricole que connait le Niger ces dernières années qui ont pour conséquence les périodes de crises alimentaires. Le niébé (Vigna unguiculata L.) est une plante annuelle qui subit les effets néfaste de la sécheresse. Au Niger, le niébé est l'une des cultures d'exportation et est la troisième culture vivrière cultivée du pays. Adapté aux conditions climatiques, édaphiques, et socio-économiques du Niger qui est le second pays producteur mondial après le Nigeria avec 1548000 tonnes en 2008 (INS, 2010), le niébé joue des multiples rôles dans l'alimentation de l'homme et du bétail de part ses graines riches en protéines et ses fanes à haute valeur fourragère. Malgré ces multiples rôles qu'il joue, force est de constater que le Niger enregistre le plus faible rendement $(210 \mathrm{~kg}$ ha-1 $^{-1}$ en moyenne de 2005 à 2007) qui est très loin du rendement moyen mondial estimé à $454 \mathrm{~kg} \mathrm{ha-1}$

\section{MATERIEL ET METHODES}

Site d'expérimental : Les essais ont été conduits dans la station expérimentale de l'ICRISAT Sadoré (Niger) située à $40 \mathrm{~km}$ au Sud-Est de la ville de Niamey entre $13^{\circ} 15^{\prime}$ de latitude Nord et $2^{\circ} 18^{\prime}$ de longitude Est. Le sol est de type alfisols à forte teneur en sable $(90 \%)$ avec les caractéristiques suivantes : $\mathrm{pH}-\mathrm{H}_{2} \mathrm{O}(5,28)$, capacité d'échange cationique $\left(1,91 \mathrm{cmol} \mathrm{kg}^{-1}\right)$, matière organique
(Abate et al., 2012). Cette faiblesse de rendement est due au fait que cette culture est confrontée à des régimes pluviométriques déficitaires qui se traduisent par des stress hydriques sévères et erratiques. Ces déficits hydriques limitent l'expression du potentiel de production des variétés. Ainsi, en agissant sur les paramètres morpho-physiologiques, la sécheresse conduit à des rendements faibles et instables (Belko et al., 2014). L'importance de l'effet d'un déficit hydrique sur le rendement final en graines dépend de stade auquel il intervient ainsi que de sa durée (Amigues et al., 2009 ; Hamidou et al., 2005). Les stades de floraison et de maturation physiologique des graines sont les plus sensibles au déficit hydrique car c'est à ces stades que l'activité photosynthétique est en faveur de la migration des assimilats des feuilles vers les graines (Hall et al., 2004). C'est ainsi que le rendement du niébé au Niger reste très loin de rendement moyen mondial. Pour tacler ce problème et parvenir à rehausser sa productivité, l'identification des variétés tolérantes et à haut rendement s'impose. Cette étude s'est fixée comme objectifs l'identification des variétés de niébé performantes pour la production de graines et tolérantes au déficit hydrique intermittent d'une part et d'investiguer les traits associés à cette tolérance d'autre part.

$(0,22 \%)$, azote total $\left(204,30 \mathrm{mg}^{-\mathrm{N} \mathrm{kg}^{-1}}\right)$, phosphore total $\left(26,25 \mathrm{mg}^{\mathrm{P} \mathrm{kg}^{-1}}\right)$, phosphore assimilable ou $\mathrm{P}$ bray1 $\left(1,83 \mathrm{mg}^{-} \mathrm{P} \mathrm{kg}{ }^{-1}\right)$.

Matériel végétal : Le matériel végétal est composé de dix-huit variétés du niébé. Les origines et les cycles de ces variétés sont consignés dans le tableau 1. 

unguiculata (L.) Walpers] en conditions du déficit hydrique

Tableau 1 : Quelques caractéristiques des 18 génotypes de niébé étudiés Table 1: Characteristics of investigated cowpea genotypes

\begin{tabular}{|c|c|c|c|c|c|}
\hline Nom & Origine & Cycle & Nom & Origine & Cycle \\
\hline $58-57$ & Sénégal & Semi-précoce & IT99K-124-5 & Nigeria & Semi-précoce \\
\hline Apagbaala & Ghana & Précoce & KVX-403 & Burkin Faso & Semi-précoce \\
\hline ITOOK-901-6 & Nigeria & Semi-précoce & KVX-414-22-2 & Burkin Faso & - \\
\hline IT90K-284-2 & Nigeria & Semi-précoce & $\mathrm{KVX}-525$ & Burkin Faso & Semi-précoce \\
\hline IT93D-442 & Nigeria & Semi-précoce & KVX-61-1 & Burkin Faso & Précoce \\
\hline IT93K-2046 & Nigeria & Précoce & Mélakh & Sénégal & Précoce \\
\hline IT96D-610 & Nigeria & Semi-précoce & Mougne & Sénégal & Semi-précoce \\
\hline IT98K-1111-1 & Nigeria & Précoce & Mouride & Sénégal & Précoce \\
\hline IT98K-128-2 & Nigeria & Semi-précoce & Yacine & Sénégal & Précoce \\
\hline
\end{tabular}

Dispositif expérimental: Le dispositif expérimental utilisé est un split plot à quatre répétitions, en grande parelle le régime hydrique et en petite parcelle la variété. L'écartement entre les répétitions est de $6 \mathrm{~m}$. Au sein de chaque répétition, il y a 18 parcelles élémentaires d'une superficie de $2 \mathrm{~m} \times 1 \mathrm{~m}=2 \mathrm{~m}^{2}$ espacées de $2 \mathrm{~m}$. L'essai a une superficie totale de $1680 \mathrm{~m}^{2}$. Un seul facteur (régime hydrique) est mis en jeu avec deux modalités : $\checkmark \quad \mathrm{Bl}$ : Bien irrigue ou traitement témoin avec une alimentation hydrique non limitant,

$\checkmark \quad \mathrm{DH}$ : Déficit hydrique intermittent ou traitement stressé.

Les parcelles soumises au déficit hydrique sont convenablement arrosées ( $30 \mathrm{~mm}$ par irrigation) comme celles du traitement témoin $\mathrm{BI}$ jusqu'au 25 ème jour après semis (JAS) où le DH a été imposé. L'application du DH a consisté à interrompre l'irrigation jusqu'à ce que la majorité des plantes montrent des symptômes nets de flétrissement avant d'être arrosées puis interruption de l'irrigation à nouveau, ce cycle se poursuit jusqu'à la maturité de gousses.

Installation de l'essai : Les expérimentations ont été réalisées du Septembre au Décembre 2014 pour l'essai conduit en saison froide et d'Avril à Juin 2015 pour l'essai mené en saison chaude. La préparation du terrain a consisté en un laboure profond $(15 \mathrm{~cm})$ suivi d'un hersage à sec. Le sol a été ensuite fertilisé avec de la fumure organique (2000 $\left.\mathrm{kg} \mathrm{ha}^{-1}\right)$ et de l'engrais 15-15-15 (en raison de $200 \mathrm{~kg} \mathrm{ha}^{-1}$ ). Une irrigation de $30 \mathrm{~mm}$ d'eau a été effectuée la veille du semis à l'aide d'un système d'irrigation Linear-move (Valmont Irrigation Inc., Valley, Nebraska, États-Unis). Chaque parcelle élémentaire comporte 2 lignes. Les écartements entre les lignes et les poquets sont respectivement de $20 \mathrm{~cm}$ et $50 \mathrm{~cm}$ soient 10 plants par ligne et 20 poquets par parcelle.

Observations et mesures : Les paramètres climatiques (températures minimales et maximales, humidités relatives minimales et maximales) ont été recueillis quotidiennement à partir d'une station météorologique située à proximité des essais. Les dates du début et de $50 \%$ floraison ont été déterminées. L'indice de la surface foliaire ou LAI (Leaf Area Index) a été mesuré pour suivre l'évolution du couvert végétal. La mesure a été effectuée entre $9 \mathrm{~h}$ et $11 \mathrm{~h} 30$ à l'aide d'un LAl-2200 (Licor, Inc., Lincoln, Nebraska 68504, USA). Dans chaque parcelle, une mesure est effectuée au-dessus du couvert et trois mesures en dessous du couvert le long des diagonales passant entre 2 lignes formées par six poquets. La série de mesures est répétée deux fois dans chaque parcelle. Dans l'expérimentation conduite en saison froide, des plants des extrémités des lignes ont été prélevés au 30 ème jour après l'imposition de stress hydrique afin de déterminer la surface foliaire (SF) à l'aide d'un planimètre leaf area meter LI-3100 (Licor Inc., Lincoln, Nebraska 68504-0425, USA). Après avoir mesuré la surface foliaire, les feuilles ont été séchées à l'étuve à $70^{\circ} \mathrm{C}$ pendant 48 heures pour déterminer leurs poids secs (PS). SF et PS ont été utilisées pour déterminer la surface foliaire spécifique ou Specific Leaf Area (SLA=SF/PS). La récolte a été faite dans un carré de rendement de $1 \mathrm{~m}^{2}$ et les gousses ont séchées au soleil. Après séchage, les gousses sont pesées puis décortiquées et les graines pesées afin de déterminer le poids. Un indice de sensibilité à la sécheresse (IS) a été utilisé pour apprécier l'effet du déficit hydrique sur le rendement (Hamidou et al., 2005) suivant la formule : IS = 1 - Rs / Rt où Rs est le rendement moyen de chaque génotype sous sécheresse et $\mathrm{Rt}$ le rendement moyen de chaque génotype en conditions d'irrigation optimale.

Analyse des données : Les données collectées ont été soumises à l'analyse de variance (ANOVA) à l'aide du logiciel GenStat 14ème édition. La comparaison des moyennes a été faite en utilisant le teste de Tukey au seuil de $5 \%$. Les graphiques sont réalisés à l'aide du logiciel de Microsoft Excel 2013. 


\section{RESULTATS}

Effet du déficit hydrique sur le stade phénologique : Le tableau 2 illustre l'influence du déficit hydrique sur la date de $50 \%$ floraison. Le déficit hydrique a retardé la date de $50 \%$ floraison de 5 jours en moyenne en contre saison froide alors qu'en contre saison chaude le régime hydrique n'a pas influencé les dates du début et de $50 \%$ floraison. En saison chaude, les variétés IT93K-2046, IT98K-1111-1, Mélakh, IT96D-610 et IT99K-124-4 sont précoces puisqu'elles ont atteint le stade de $50 \%$ floraison à moins de $50 \mathrm{JAS}$ comparativement aux variétés IT93D-442, IT98K-128-2 et KVX-414-22-2 dontle stade $50 \%$ été observé au dela de $60 \mathrm{JAS}$.

Effets du déficit hydrique sur les paramètres morphophysiologiques

Indice de la surface foliaire : L'analyse de la variance de l'indice de la surface foliaire a montré de différence significative $(P<0,001)$ entre les variétés et les régimes hydriques. Le stress hydrique a significativement réduit l'indice de la surface foliaire ou LAI (Figure 1). Le LAI a connu des réductions de $15 \%$ en saison froide et de $48 \%$ en saison chaude. Les analyses statistiques ont révélé que les valeurs de cet indice sont plus élevées en saison chaude (3,58 et 1,87 pour Bl et DH respectivement) qu'en saison froide (2,06 et 1,75 pour $\mathrm{Bl}$ et $\mathrm{DH}$ respectivement). Surface foliaire, poids sec des feuilles et surface foliaire spécifique en saison froide : Le déficit hydrique a diminué la surface foliaire de $30 \%$ en moyenne (Figure 2). Les variétés les plus affectées sont IT96D-610 (56\%), KVX-414-22-2 (49\%), Apagbaala (45\%), Mouride (43\%) et IT93D-442 (40\%). A l'instar de la surface, les plantes soumises au déficit hydrique ont un poids sec des feuilles plus faibles $\left(10,54 \mathrm{~g} \mathrm{plant}^{-1}\right)$ par rapport à celles du traitement bien irrigué (14,38 g plant $\left.{ }^{-1}\right)$ (Figure 3). Les variétés KVX-61-1, IT96D-610 et Apagbaala se révèlent plus sensibles au déficit hydrique avec respectivement 56 , 51 et $50 \%$ de réduction du poids sec des feuilles. L'analyse de la variance de la surface foliaire spécifique a montré une différence significative entre les variétés $(P=0,001)$ et un effet significatif du régime hydrique $(P=0,018)$. Les variétés Mougne, Mouride, KVX-525, KVX-403 et IT93D-442 ont été les plus affectées par le déficit hydrique (Figure 4).

Tableau 2 : effets de traitement hydrique et de la période sur les dates du début et $50 \%$ floraison chez les 18 génotypes évalués. $\mathrm{BI}=$ traitement hydrique bien irrigué $\mathrm{DH}=$ traitement déficit hydrique; $\mathrm{SF}=\mathrm{Saison}$ froide ou essail; $\mathrm{SC}=$ Saison chaude ou essai2

Table 2: water treatment and period effects on beginning and $50 \%$ flowering dates for 18 cowpea genotypes. BI $=$ water well treatment $\mathrm{DH}=$ water deficit treatment, $\mathrm{SF}=$ cold season or trial1, $\mathrm{SC}=$ Hot season or trial 2

\begin{tabular}{|c|c|c|c|c|c|c|c|c|}
\hline \multirow{3}{*}{ Génotypes } & \multicolumn{4}{|c|}{ Début floraison } & \multicolumn{4}{|c|}{$50 \%$ floraison } \\
\hline & \multicolumn{2}{|c|}{ BI } & \multicolumn{2}{|c|}{ DH } & \multicolumn{2}{|c|}{ BI } & \multicolumn{2}{|c|}{ DH } \\
\hline & SF & SC & SF & SC & SF & $\mathrm{SC}$ & SF & sc \\
\hline $58-57$ & 50 & 43 & 58 & 38 & 53 & 51 & 60 & 43 \\
\hline Apagbaala & 56 & 39 & 55 & 39 & 59 & 49 & 63 & 48 \\
\hline IT00K-901-6 & 57 & 57 & 40 & 51 & 63 & - & 76 & 53 \\
\hline IT90K-284-2 & 60 & 40 & 61 & 41 & 65 & 50 & 66 & 47 \\
\hline IT93D-442 & 57 & 51 & 63 & 56 & 62 & 63 & 71 & 66 \\
\hline IT93K-2046 & 59 & 39 & 61 & 36 & 63 & 43 & 67 & 42 \\
\hline IT96D-610 & 56 & 43 & 57 & 44 & 61 & 48 & 60 & 48 \\
\hline IT98K-1111-1 & 55 & 34 & 55 & 34 & 64 & 40 & 63 & 39 \\
\hline IT98K-128-2 & 59 & 51 & 61 & 54 & 64 & 61 & 66 & 63 \\
\hline IT99K-124-5 & 58 & 35 & 57 & 36 & 62 & 46 & 68 & 42 \\
\hline KVX-403 & 56 & 52 & 59 & 52 & 61 & 58 & 68 & 58 \\
\hline KVX-414-22-2 & 53 & 48 & 59 & 50 & 57 & 64 & 67 & - \\
\hline KVX-525 & 49 & 47 & 58 & 49 & 53 & 55 & 63 & 54 \\
\hline KVX-61-1 & 53 & 44 & 56 & 45 & 58 & 52 & 63 & 52 \\
\hline Mélakh & 58 & 41 & 58 & 38 & 62 & 48 & 67 & 47 \\
\hline Mougne & 54 & 43 & 60 & 40 & 59 & 49 & 64 & 53 \\
\hline Mouride & 56 & 39 & 60 & 38 & 60 & 48 & 66 & 48 \\
\hline Yacine & 61 & 43 & 60 & 40 & 70 & 54 & 63 & 57 \\
\hline Moyenne & 55 & 45 & 58 & 46 & 61 & 52 & 66 & 52 \\
\hline
\end{tabular}




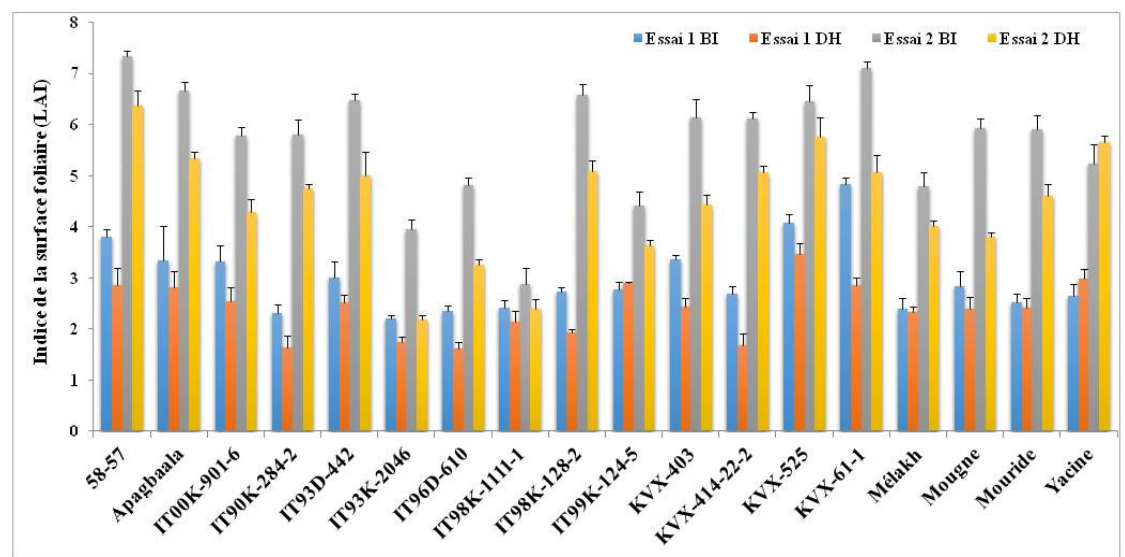

Figure 1: Effets de déficit hydrique sur l'indice de la surface foliaire (LAI) en saison froide (Essai 1) et en saison chaude (Essai 2). $\mathrm{BI}=$ traitement hydrique bien irrigué; $\mathrm{DH}=$ traitement déficit hydrique

Figure 1: Water deficit effects on leaf area index (LAI) during cold season (Trial 1) and hot season (Trial

2). $\mathrm{BI}=$ well watered treatment $; \mathrm{DH}=$ water deficit treatment

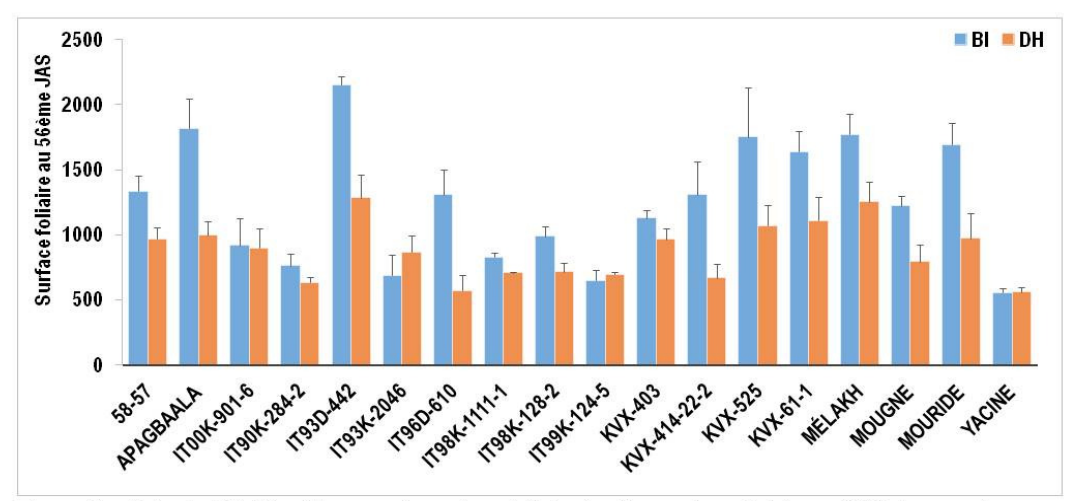

Figure 2: effets de déficit hydrique sur la surface foliaire $\left(\mathrm{cm}^{2}\right)$ en saison froide au $30^{\text {ème jour après }}$ imposition du stress (JAIS). $\mathrm{BI}=$ traitement hydrique bien irrigué; $\mathrm{DH}=$ traitement déficit hydrique.

Figure 2: water deficit effect on leaf area per plant $\left(\mathrm{m}^{2}\right)$ during cold season (trial 1 ) $30^{\text {th }}$ day after stress imposition(JAIS). BI = well-watered treatment; $\mathrm{DH}=$ water-stress treatment.

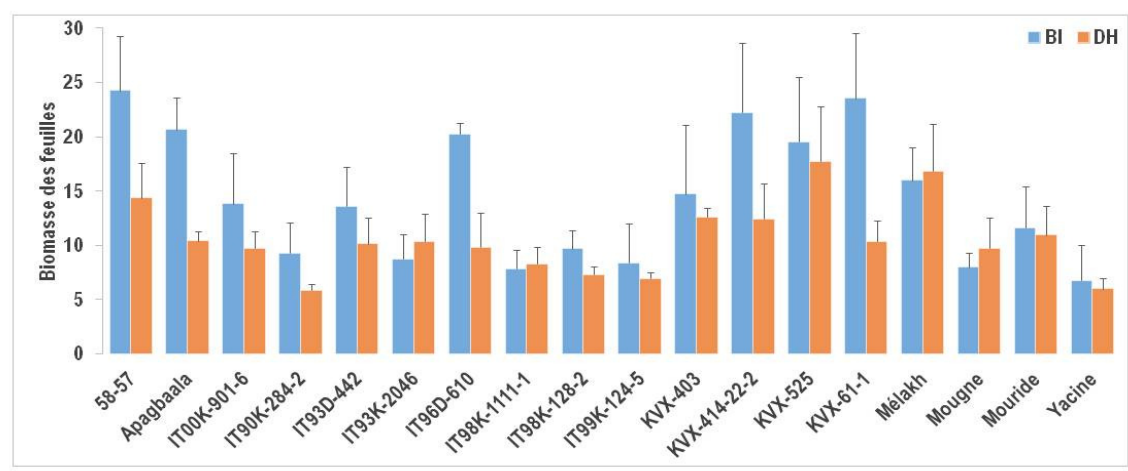

Figure 3 : effets de déficit hydrique sur la biomasse des feuilles par plant (en g) en saison froide au $30^{\text {ème jour }}$ après imposition du stress (JAIS). BI= traitement hydrique bien irrigué; $\mathrm{DH}=$ traitement déficit hydrique Figure 3: water deficit effect on leaf biomass per plant $(\mathrm{g})$ during cold season (trial 1 ) $30^{\text {th }}$ day after stress imposition (JAIS). BI = well-watered treatment; $\mathrm{DH}=$ water-stress treatment. 


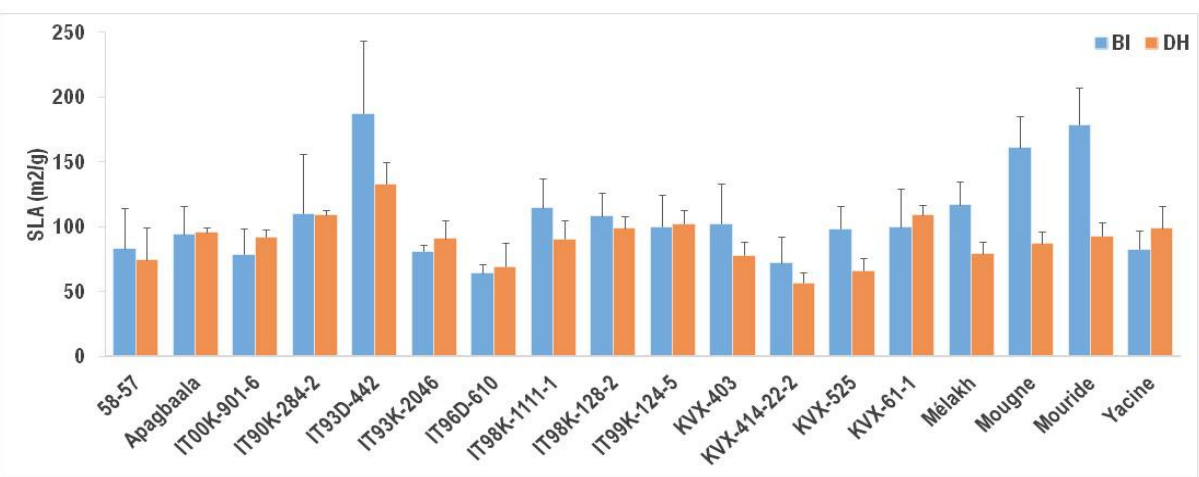

Figure 4 : effets du déficit hydrique sur la surface foliaire spécifique par plant $\left(\mathrm{m}^{2} \mathrm{~g}^{-1}\right)$ en saison froide au $30^{\mathrm{ème}}$ jour après imposition du stress (JAIS). $\mathrm{Bl}=$ traitement hydrique bien irrigué; $\mathrm{DH}=$ traitement déficit hydrique Figure 4: water deficit effect on specific leaf area per plant $\left(\mathrm{m}^{2} \mathrm{~g}^{-1}\right)$ during cold season (trial 1 ) $30^{\text {th }}$ day after stress imposition $(\mathrm{JAIS})$. BI = well-watered treatment; $\mathrm{DH}=$ water-stress treatment.

Effet du régime hydrique sur les variétés et le rendement graines : L'effet du déficit hydrique sur le rendement graines en saison froide et en saison chaude est présenté aux Figures figures 5 et 6 .. Les analyses des résultats ont révélé une interaction significative $(P<0,001)$ entre les variétés, le régime hydrique et la saison. Ainsi en saison froide, les variétés qui ont présenté les rendements graines les plus élevés sous une irrigation optimale sont KVX-61-1 (3001 kg ha-1), KVX-403 (2046 $\mathrm{kg} \mathrm{ha}^{-1}$ ), Mougne (1682 kg ha-1), KVX-525 (1609 kg ha-1), IT93D-442 (1594 kg ha-1) et KVX-414-22-2 (1562 kg ha-1) alors que Yacine (311 kg ha-1) et IT00K-901-6 (501 kg ha1) ont présenté les plus faibles rendements. Le déficit hydrique a réduit le rendement graines de $88 \%$. Les rendements les plus élevés en conditions de deficit hydrique ont été enregistrés chez les variétés IT96D-610 (355 kg ha-1), IT98K-1111-1 (331 kg ha-1), KVX-414-22-2
(278 kg ha-1), 58-57 (271 kg ha-1) et KVX-61-1 (266 kg ha1). Ses variétés se révèlent tolérantes d'au déficit hydrique en saison froide. En saison chaude, les meilleurs rendements graines sous traitement bien irrigué ont été observés chez les variétés KVX-525 $(2018 \mathrm{~kg}$ ha1), 58-57 (1989 kg ha-1), IT96D-610 (1944 kg ha-1), Mougne (1596 kg ha-1), KVX-61-1 (1388 kg ha-1) et IT98K-1111-1 (1360 kg ha-1) tandis que les variétés KVX414-22-2 (289 kg ha-1), IT93D-442 (353 kg ha-1) et Apagbaala $\left(480 \mathrm{~kg} \mathrm{ha}^{-1}\right)$ ont montre des faibles rendements. Au cours de cette saison, le déficit hydrique a réduit le rendement graines de $67 \%$. Les variétés qui se sont révélé plus tolérantes en montrant les rendements graines les plus élevés sont KVX-525 (743 kg ha-1), KVX61-1 (724 kg ha-1), IT96D-610 (695 kg ha-1), Mougne (615 $\mathrm{kg} \mathrm{ha}^{-1}$ ) et IT98K-1111-1 (551 kg ha-1).

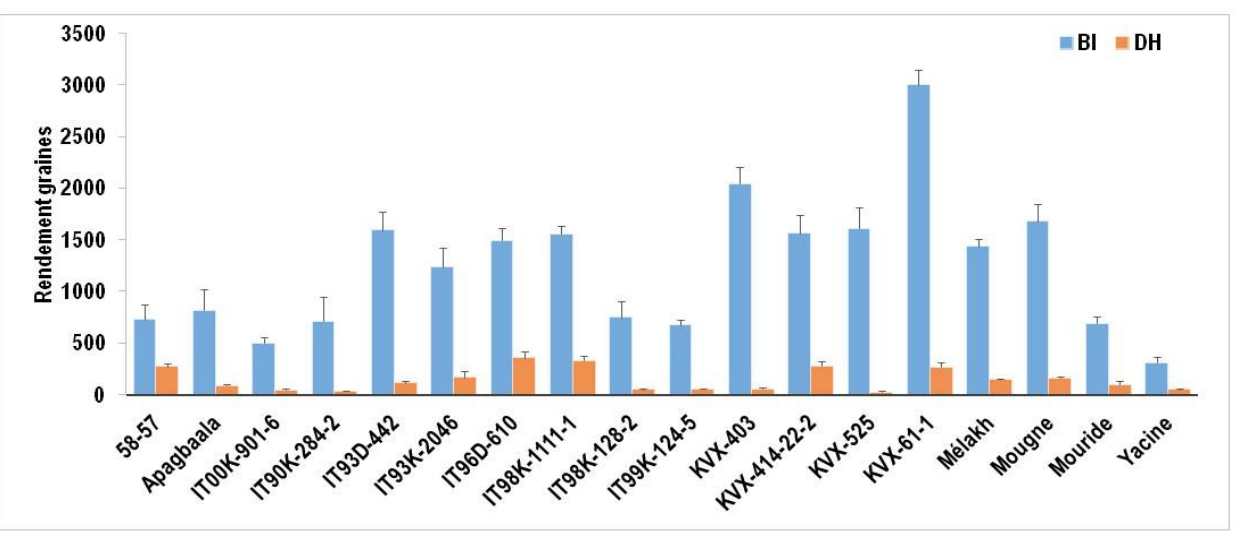

Figure 5: Rendement moyen de graines $\left(\mathrm{kg} \mathrm{ha}^{-1}\right)$ au champ en saison froide (essai 1) chez les 18 génotypes du niébé en conditions bien irrigée (BI) et de déficit hydrique (DH).

Figure 5: Seeds yield $\left(\mathrm{kg} \mathrm{ha}^{-1}\right)$ in field conditions during cold season (trial 1) for 18 cowpea genotypes under well-watered (BI) and water-stress conditions (DH). 


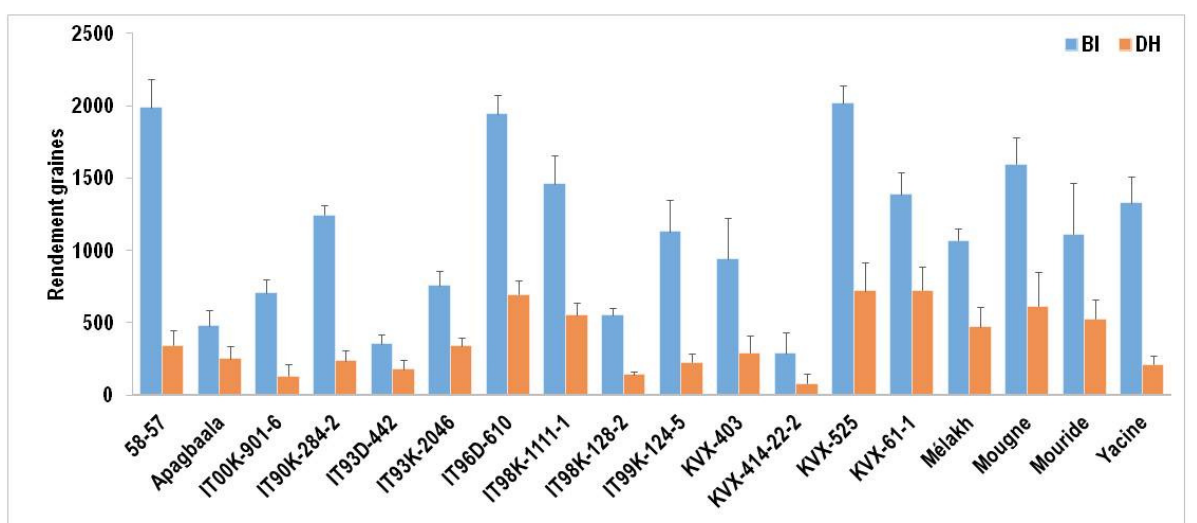

Figure 6: rendement moyen de graines (en $\mathrm{kg} \mathrm{ha}^{-1}$ ) au champ en saison chaude (essai 2) chez les 18 génotypes du niébé au champ en conditions de bonne irrigation (BI) et de déficit hydrique $(\mathrm{DH})$.

Figure 6: seeds yield $\left(\mathrm{kg} \mathrm{ha}^{-1}\right)$ in field conditions during hot season (trial 2) for 18 cowpea genotypes under well-watered (BI) and water stress conditions (DH)

\section{DISCUSSION}

Le déficit hydrique imposé a négativement affecté l'ensemble des paramètres étudiés. Les valeurs de l'indice de la surface foliaire (LAl) ont subi des baisses de $15 \%$ et $48 \%$ au 20 ème jour après imposition du stress (JAIS) respectivement en saison froide et en saison chaude. Outre la réduction de l'indice de la surface foliaire, il a été observé en saison froide des réductions moyennes de $32 \%$ de la surface foliaire spécifique (SLA). II a été démontré que lorsque les plantes sont soumises à un stress hydrique, elles adoptent des stratégies leur permettant de maintenir leur état hydrique (Passioura et Angus, 2010). C'est la stratégie consistant à fermer les stomates et à réduire la surface transpirante via la diminution de vitesse de croissance chez les plantes stressées. Cependant, cette fermeture des stomates a comme conséquence la réduction de l'activité photosynthétique (Agbicodo et al., 2009) qui impact sur le rendement et ses composantes. Les effets dépressifs du déficit hydrique sur les composantes de rendement ont été observes dans cette étude. Le stress hydrique en affectant la croissance et le développement des plantes, a réduit les rendements graines de $88 \%$ en saison froide et de $67 \%$ en saison chaude. Ces résultats corroborent ceux des travaux antérieurs sur le niébé (Aziadekey et al., 2014). Halime et al. (2014) ont rapporté que chez les plantes soumises au stress hydrique durant les stades floraison et le remplissage des gousses, la réduction de rendements était supérieure de $60 \%$ comparée aux plantes témoins. Selon Chiulele et al. (2011), la sécheresse durant les stades floraison et remplissage des gousses a le plus grand impact car elle influe directement sur le rendement. La sévérité de ce type de stress est due au fait qu'il touche les facteurs en rapport avec la formation des graines dont la photosynthèse et la translocation des assimilats (Aziadekey et al., 2014). La variation génotypique a révélé que parmi les variétés étudiées, KVX-61-1, IT96D-610 et IT98K-1111-1 ont donné les rendements moyens en graines les plus élevés quel que soit le régime hydrique et la saison de culture. Ces résultats corroborent ceux observés par Belko et al., ( 2012 ; 2014)qui ont montré que KVX-61-1 et IT96D-610 sont des variétés performantes en conditions optimales et du déficit hydrique tandis que la variété IT98K-1111-1 n'est performante que sous déficit hydrique. Le rendement élevé de KVX-61-1 peut être lié à sa capacité à poursuivre ses activités physiologiques notamment la transpiration et la photosynthèse même en conditions du déficit hydrique. Ce génotype a montré des valeurs élevées de l'indice de la surface foliaire sous les deux régimes hydriques. Ceci aurait permis à ce génotype une forte transpiration et donc une plus grande absorption de l'eau par les racines et une forte capacité photosynthétique. Somé et al., (2014) et Kihindo et al., (2016) ont observé un faible niveau du potentiel hydrique foliaire en conditions du déficit hydrique chez KVX-61-1. Ce qui suggère un ajustement osmotique des cellules dans le but de continuer à prélever le peu d'eau disponible dans les couches profonde du sol, afin de poursuivre les activités physiologiques en dépit de la contrainte hydrique du sol. Quant aux variétés IT96D-610 et IT98K-1111-1, leur performance peut s'expliquer par une floraison précoce et groupée. Les travaux de Naima (2011) ont montré que le rendement en graines est positivement corrélé à la date de floraison. Selon cet auteur, les variétés précoces ont une meilleure productivité par rapport aux variétés tardives. Ces variétés utiliseraient les assimilats photosynthétiques pour la formation des organes reproductifs (gousses et 

unguiculata (L.) Walpers] en conditions du déficit hydrique

graines) au détriment des organes végétatifs. L'étude de l'interaction entre variété et saison de la culture a montré que les variétés IT93D-442 et KVX-414-22-2 sont performantes en saison froide dans les deux régimes hydriques alors que les variétés 58-57, KVX-525, Mougne, Mouride et IT99K-124-5 se sont révélés

\section{CONCLUSION}

L'étude a montré que le déficit hydrique durant les stades floraison et formation des gousses a négativement affecté la croissance, le développement et le rendement de la culture du niébé. Les résultats obtenus ont révélé l'existence d'une variation génotypique dans la réponse à cette contrainte. II est ressorti de cette étude que la perte du rendement des graines due au déficit hydrique peut aller à plus de $88 \%$ selon sa sévérité. Sur la base des rendements graines enregistrés en conditions d'irrigation

\section{REMERCIEMENTS}

Ces travaux ont été réalisés dans le cadre d'une thèse dont la bourse est financée par le Programme Productivité Agricole en Afrique de l'Ouest (PPAAO). Les auteurs adressent aussi leurs sincères remerciements au Centre Ouest Africain de Recherche et de Services Scientifiques sur le Changement Climatique et I'Utilisation

\section{REFERENCES}

Abate T, Alene AD, Bergvinson D, Shiferaw B, Silim S, Orr A, Asfaw S, 2012. Tropical grain legumes in Africa and south Asia: knowledge and opportunities. International Crops Research Institute for the Semi-Arid Tropics.

Agbicodo EM, Fatokun CA, Muranaka S, Visser RGF, 2009. Breeding drought tolerant cowpea: constraints, accomplishments, and future prospects. Euphytica, 167(3), 353-370.

Amigues JP, Debaeke P, Itier B, Lemaire G, Seguin B, Tardieu F, Thomas A, 2006. Sécheresse et agriculture. Réduire la vulnérabilité de l'agriculture à un risque accru de manque d'eau. Expertise scientifique collective, synthèse du rapport, INRA (France).

Aziadekey M, Atayi A, Odah K, Magamana AE, 2014. Étude de l'influence du stress hydrique sur deux lignées de niébé. European Scientific Journal, ESJ, 10(30).

Belko N, Cisse N, Diop NN, Zombre G, Thiaw S, Muranaka S, Ehlers J, 2014. Selection for post flowering drought resistance in short-and medium-duration cowpeas using stress tolerance indices. Crop Science, 54(1), 25-33. meilleures sous les régimes hydriques en saison chaude. Les rendements élevés obtenus en conditions du deficit hydrique chez ces variétés en saison chaude pourraient s'expliquer par une bonne capacité de reprise de croissance et de la floraison après la levée du déficit hydrique.

non limitant et du déficit hydrique, l'étude a révélé que les variétés KVX-61-1, IT96D-610 et IT98K-1111-1 peuvent être cultivés pendant toutes les périodes de l'année et sont tolérantes à la sécheresse. Quant aux variétés IT93D-442 et KVX-414-22-2, elles se sont révélées performants en saison froide alors que 58-57, KVX-525, Mougne, Mouride et IT99K-124-5 préfèrent la saison chaude.

Adaptée des Terres (WASCAL) pour l'octroi d'une bourse partielle pour finaliser les travaux de la thèse. Ils remercient également tous ceux qui ont participé à ces travaux particulièrement $\mathrm{Dr}$ HALILOU Oumarou, $\mathrm{Mr}$ BACHAROU Falké Achirou, Mme HEYNIKOYE Tahirou Mariama et Mr HALILOU Hayyo.

Belko N, Zaman-Allah M, Cisse N, Diop NN, Zombre G, Ehlers JD, Vadez V, 2012. Lower soil moisture threshold for transpiration decline under water deficit correlates with lower canopy conductance and higher transpiration efficiency in droughttolerant cowpea. Functional Plant Biology, 39(4), 306-322.

Benoît S, Diouf O, Diouf M, Harold RM, Casimir B, 2002. Utilisation de paramètres agronomiques comme critères de résistance à la sécheresse chez trois variétés de niébé cultivées au Sénégal et au Niger. Science et changements planétaires/Sécheresse, 12(4), 259-66.

Chiulele RM, Mwangi G, Tongoona P, Ehlers JD Ndeve $A D, 2011$. Assessment of cowpea genotypes for variability to drought tolerance. In 10th African Crop Science Conference Proceedings, Maputo, Mozambique, 10-13 October 2011 (pp. 531537). African Crop Science Society.

Clavel D, Drame NK, Diop ND, Zuily-Fodil Y, 2005. Adaptation à la sécheresse et création variétale : le cas de l'arachide en zone sahéliennePremière partie: revue 
bibliographique. Oléagineux, Corps gras, Lipides, 12(3), 248-260.

INS, 2010. Institut National de la Statistique. Annuaire statistique des cinquante ans d'indépendance du Niger, 330p.

Dugje IY, Omoigui LO, Ekeleme F, Kamara AY, Ajeigbe $H, 2009$. Production du niébé en Afrique de I'Ouest : Guide du paysan. IITA, Ibadan, Nigeria.

FAOSTAT, 2014. Food and Agricultural Organization of the United Nations (FAO) Statistics.

Naima G, 2011. Étude de la diversité génétique de quelques écotypes locaux de Vigna unguiculata (L.) Walp. Cultivés en Algérie (Doctoral dissertation). École Normale Supérieure Agronomique (ENSA) El Harrach - Alger. 188p.

Halilou O, Hamidou F, Taya BK, Mahamane S, Vadez V, 2015. Water use, transpiration efficiency and yield in cowpea (Vigna unguiculata) and peanut (Arachis hypogaea) across water regimes. Crop and Pasture Science, 66(7), 715-728.

Halime MH, BelkoN, Cisse N, Sine B, Ndoye I, 2014. Amélioration de l'adaptation à la sécheresse chez le niébé (Vigna unguiculata L. Walpers). Journal of Applied Biosciences, 77(1), 6550-6563.

Hall AE, 2004. Breeding for adaptation to drought and heat in cowpea. European Journal of Agronomy, 21(4), 447-454.

Hamidou F, 2006. Paramètres physiologiques, biochimiques et agronomiques pertinents pour l'amélioration de l'adaptation du niébé (Vigna unguiculata (L.) Walp.) au déficit hydrique (Doctoral dissertation). Université de Ouagadougou, 168p.

Hamidou F, Dicko MH, Zombre G, Traoré AS, Guinko S, 2005. Réponse adaptative de deux variétés de niébé à un stress hydrique. Cahiers Agricultures, 14(6), 561-567.

Nana R, Tamini Z, Sawadogo M, 2009. Effets d'un stress hydrique intervenu pendant le stade végétatif et la phase de floraison chez le gombo. International Journal of Biological and Chemical Sciences, 3(5).

Ogbonnaya Cl, Sarr B, Brou C, Diouf O, Diop NN, RoyMacauley H, 2003. Selection of cowpea genotypes in hydroponics, pots, and field for drought tolerance. Crop Science, 43(3), 11141120.

Okon JE, 2013. Effect of water stress on some growth aspects of two varieties of cowpea, Vigna unguiculata (L.) Walp Fabaceae. Bulletin of
Environment, Pharmacology and Life Sciences, 2(5), 69-74.

Passioura JB, Angus JF, 2010. Improving productivity of crops in water-limited environments. Advances in agronomy, 106, 37-75.

Toudou A, 1995. Étude de deux parasites d'origine tellurique sur le niébé: Macrophomina phaseolina (Tassi) GOID. Et Striga gesneriö̈des (Willd) VATKE (Doctoral dissertation). Université Abdou Moumouni de Niamey, 102p.

Somé PP, Hien E, Tozo K, Zombré G, et Dianou D, 2014. Effets de six composts sur les réponses physiologiques, biochimiques et agronomiques du niébé Vigna unguiculata $L$. Walp var. KVX61.1 au déficit hydrique. IJBCS. 1:31-45.

Kihindo AP, Bazie HR, Ouedraogo RF, Some PP, Dondasse E, Zombre G, Tozo K, 2016. Variation de la réponse physiologique et agronomique du niébé (Vigna unguiculata L. Walp) à différentes dates de semis au Burkina Faso. Journal of Applied Biosciences, 107(1), 10353-10361. 